\title{
Exact Elliptic Solution for Non-Linear Klein-Gordon Equation Via Auxiliary Equation Method
}

\author{
G. M. Moatimid, M. H. M. Moussa, Rehab M. El-Shiekh, A. A. El-Satar*
}

Department of Mathematics, Faculty of Education, Ain Shams University, Roxy, Hiliopolis, Cairo, Egypt

\begin{abstract}
By using symbolic computation, we apply Auxiliary equation method to construct exact solutions of Non-Linear Klein-Gordon equation. We show that Auxiliary equation method provides a powerful mathematical tool for solving nonlinear evolution equations in mathematical physics.
\end{abstract}

Keywords Traveling Wave, Exact Solutions And Auxiliary Equation

\section{Introduction}

The investigation of the travelling wave solutions for non-linear partial differential equations plays an important role in the study of non-linear physical phenomena. Non-linear wave phenomena appears in various scientific and engineering fields, such as fluid mechanics, plasma physics, optical fibers, biology, solid state physics, chemical kinematics, chemical physics and geochemistry. Non-linear wave phenomena of dispersion, dissipation, diffusion, reaction and convection are very important in non-linear wave equations. In recent years, new exact solutions may help to find new phenomena. A variety of powerful methods, such as inverse scattering method[1,2], bilinear transformation[3], the tanh-sech method[4-6], extended tanh method[7,8], sine-cosine method[9,10], homogeneous balance method[11], Exp-function method[12,13], improved tanh-function method[14] and Auxiliary equation method[15] were used to develop non-linear dispersive and dissipative problems.

\section{Auxiliary Equation Method}

Consider a given nonlinear wave equation

$$
N\left(u, u_{t}, u_{x}, u_{x x}, u_{x x}, u_{t t}, \ldots\right)=0
$$

we seek its wave solutions.

$$
\begin{aligned}
& u=U(\eta), \quad v=V(\eta), \\
& \eta=k_{1} x+k_{2} y+k_{3} z-w t .
\end{aligned}
$$

Consequently, (1.1) is reduced to the ordinary differential equation (ODE):

$$
U\left(u, w u^{\prime}, u^{\prime}, u^{\prime \prime}, \ldots\right)=0
$$

* Corresponding author:

a_a_elsatar@yahoo.com (A. A. El-Satar)

Published online at http://journal.sapub.org/am

Copyright (C) 2012 Scientific \& Academic Publishing. All Rights Reserved
Auxiliary equation method is based on the assumption that the travelling wave solutions can be expressed in the following form

$$
u(\eta)=\sum_{i=0}^{\infty} a_{i}[\varphi(\eta)]^{i}
$$

where $\varphi(\eta)$ satisfies Auxiliary equation method

$$
\varphi^{\prime 2}(\eta)=R+\mathrm{Q} \varphi^{2}(\eta)+P \varphi^{4}(\eta) .
$$

\section{Non-Linear Klein-Gordon Equation}

We study the following well-known the nonlinear Klein-Gordon equation:

$$
u_{t t}-u_{x x}-u_{y y}-u_{z z}+c u-r|u|^{2} u=0,
$$

for $c \succ 0, r \prec 0$.

To Higgs equation and for $\mathrm{c}=0$, it assumes the form of Yang-Milles equation. The Klein- Gordon equation has been studied in many literatures, e.g.,[16-18]. However, numerical treatment for Klein--Gordon equation is rarely reported (cf.[19-21]). Particularly,[20] concern with the decomposition method and difference method used in[21] nonlinear problems. We take $\mathrm{u}=\mathrm{U}+\mathrm{i} \mathrm{V}$, then by used he transformation (2.2) we get:

$$
\begin{aligned}
& \left(w^{2}-k_{1}^{2}-k_{2}^{2}-k_{3}^{2}\right) U^{\prime \prime}+c U-r\left(U^{3}+V^{3} U\right)=0, \\
& \left(w^{2}-k_{1}^{2}-k_{2}^{2}-k_{3}^{2}\right) V^{\prime \prime}+c V-r\left(V^{3}+U^{3} V\right)=0 .
\end{aligned}
$$

Now balancing $\mathrm{U}^{\prime \prime}$ with $\mathrm{U}^{3}$ and $\mathrm{V}^{\prime \prime}$ with $\mathrm{U}^{3} \mathrm{~V}$ gives $\mathrm{M}=1$, $\mathrm{N}=1$. Therefore we may choose:

$$
\begin{aligned}
& u(\eta)=a_{0}+a_{1} \varphi+a_{2} \varphi^{2}, \\
& v(\eta)=b_{0}+b_{1} \varphi+b_{2} \varphi^{2} .
\end{aligned}
$$

Substituting from equations (3.3) in equation (3.1), collect the coefficients of $\varphi(\eta)^{i}(i=0, \ldots, 3)$ and equated them to zero we obtain the system 


$$
\begin{aligned}
& \mathrm{rb}_{2}^{3}+r b_{2} a_{2}^{2}=0 \text {, } \\
& a_{2}^{3}+r a_{2} b_{2}^{2}=0 \text {, } \\
& 3 r b_{1} b_{2}^{2}+r b_{1} a_{2}^{2}+2 r b_{2} a_{1} a_{2}=0, \\
& \mathrm{ra}_{0} \mathrm{~b}_{2}^{2}+2 \mathrm{ra}_{1} \mathrm{~b}_{1} \mathrm{~b}_{2}+\mathrm{ra}_{2}\left(2 \mathrm{~b}_{0} \mathrm{~b}_{2}+\mathrm{b}_{1}^{2}\right)=0 \text {, } \\
& 2 \mathrm{ra}_{2} \mathrm{~b}_{0} \mathrm{~b}_{1}+\mathrm{r}\left(4 \mathrm{a}_{0} \mathrm{a}_{1} \mathrm{a}_{2}+\mathrm{a}_{1}\left(2 \mathrm{a}_{0} \mathrm{a}_{2}+\mathrm{a}_{1}^{2}\right)\right)=0 \text {, } \\
& 2\left(\mathrm{a}_{1}^{2} \mathrm{a}_{0}+\mathrm{a}_{2} \mathrm{a}_{0}^{2}\right)+4\left(\mathrm{w}^{2}-\mathrm{k}_{1}^{2}-\mathrm{k}_{2}^{2}-\mathrm{k}_{3}^{2}\right) \mathrm{aQ}=0, \\
& 2\left(w^{2}-k_{1}^{2}-k_{2}^{2}-k_{3}^{2}\right) a_{2} R+c a-r a_{0} b_{2}-r_{0}^{3}=0 \text {, } \\
& 2 \mathrm{rb}_{2} \mathrm{a}_{0} \mathrm{a}_{1}+\mathrm{r}\left(4 \mathrm{~b}_{0} \mathrm{~b}_{1} \mathrm{~b}_{2}+\mathrm{b}_{1}\left(2 \mathrm{~b}_{0} \mathrm{~b}_{2}+\mathrm{b}_{1}^{2}\right)\right)=0 \text {, } \\
& 6\left(w^{2}-k_{1}^{2}-k_{2}^{2}-k_{3}^{2}\right) b_{2} P-r\left(b_{0} b_{2}^{2}+2 b_{1}^{2} b 2=0,\right. \\
& 2\left(\mathrm{w}^{2}-\mathrm{k}_{1}^{2}-\mathrm{k}_{2}^{2}-\mathrm{k}_{3}^{2}\right) \mathrm{b}_{2} \mathrm{R}+\mathrm{cb}_{0}-\mathrm{rb}_{0} \mathrm{a}_{0}^{2}-\mathrm{rb}_{0}^{3}=0 \text {, } \\
& \left.b_{2}\left(2 b_{0} b_{2}+b_{1}^{2}\right)\right)-r b_{0} a_{2}^{2}-2 r b_{1} a_{1} a_{2}-r b_{2} \\
& \left(2 \mathrm{a}_{0} \mathrm{a}_{2}+\mathrm{a}_{1}^{2}\right)=0 \text {, } \\
& 2 \mathrm{ra}_{0} \mathrm{~b}_{0} \mathrm{~b}_{1}-\mathrm{ra}_{1} \mathrm{~b}_{0}^{2}+\left(\mathrm{w}^{2}-\mathrm{k}_{1}^{2}-\mathrm{k}_{2}^{2}-\mathrm{k}_{3}^{2}\right) \mathrm{a}_{1} \mathrm{Q} \\
& +\mathrm{ca}_{1}-3 \mathrm{ra}_{0}^{2} \mathrm{a}_{1}=0 \text {, } \\
& 2 \mathrm{ra}_{0} \mathrm{~b}_{0} \mathrm{~b}_{1}+\mathrm{ra}_{1} \mathrm{~b}_{0}^{2}-\left(\mathrm{w}^{2}-\mathrm{k}_{1}^{2}-\mathrm{k}_{2}^{2}-\mathrm{k}_{3}^{2}\right) \mathrm{a}_{1} \mathrm{Q} \\
& -\mathrm{ca}_{1}+3 \mathrm{ra}_{0}^{2} \mathrm{a}_{1}=0 \text {, } \\
& c a_{2}-r_{0}\left(2 b_{0} b_{2}+b_{1}^{2}\right)-2 r_{1} b_{0} b_{1}-r a_{2} b_{0}^{2} \\
& -\mathrm{ra}_{0}\left(2 \mathrm{a}_{0} \mathrm{a}_{2}+\mathrm{a}_{1}^{2}\right)=0 \text {, } \\
& 6\left(w^{2}-k_{1}^{2}-k_{2}^{2}-k_{3}^{2}\right) a_{2} P-r\left(a_{0} a_{2}^{2}+2 a_{1}^{2} a_{2}\right. \\
& \left.+\mathrm{a}_{2}\left(2 \mathrm{a}_{0} \mathrm{a}_{2}+\mathrm{a}_{1}^{2}\right)\right)=0 \text {. }
\end{aligned}
$$

Solving the system of algebraic equations with the aid of Maple, in Eq.(3.3), we obtain the following results:

$$
\begin{aligned}
& a_{0}=a_{2}=b_{0}=b_{2}=0, \\
& a_{1}^{2}+b_{1}^{2}=\frac{-2 c P}{r \mathrm{Q}}, \\
& c=-\left(\mathrm{w}^{2}-\mathrm{k}_{1}^{2}-\mathrm{k}_{2}^{2}-\mathrm{k}_{3}^{2}\right) \mathrm{Q},
\end{aligned}
$$

Substituting these results into (3.3) and with the aid of Appendix A, we obtain the following multiple soliton-like and triangular periodic solutions for (DSW) system:

$$
\begin{gathered}
U_{1}=\sqrt{\frac{2 c m^{2}}{r\left(1+m^{2}\right)}-b_{1}^{2}} \operatorname{sn}(\eta), V_{1}=b_{1} \operatorname{sn}(\eta) . \\
U_{2}=\sqrt{\frac{2 c m^{2}}{r\left(m^{2}-1\right)}-b_{1}^{2}} \operatorname{sn}(\eta), V_{2}=b_{1} \operatorname{sn}(\eta) . \\
U_{3}=\sqrt{\frac{2 c}{r\left(2-m^{2}\right)}-b_{1}^{2}} d n(\eta), V_{3}=b_{1} d n(\eta) . \\
U_{4}=\sqrt{\frac{2 c}{r\left(1-2 m^{2}\right)}-b_{1}^{2}} d s(\eta), V_{4}=b_{1} d s(\eta) . \\
U_{5}=\sqrt{\frac{2 c}{r\left(m^{2}-2\right)}-b_{1}^{2}} \operatorname{cs}(\eta), V_{5}=b_{1} \operatorname{cs}(\eta) . \\
U_{r\left(2-m^{2}\right)}-b_{1}^{2}\left(\frac{s n(\eta)}{1 \pm d n(\eta)}\right), V_{6}=b_{1}\left(\frac{s n(\eta)}{1 \pm d n(\eta)}\right) . \\
U_{7}=\sqrt{\frac{c m^{2}}{r\left(2-m^{2}\right)}-b_{1}^{2}(\operatorname{sn}(\eta) \pm i \operatorname{cn}(\eta))}, \\
V_{7}=b_{1}(\operatorname{sn}(\eta) \pm i \operatorname{cn}(\eta)) .
\end{gathered}
$$

$$
\begin{gathered}
U_{8}=\sqrt{\frac{c}{r\left(2 m^{2}-1\right)}-b_{1}^{2}}\left(\frac{s n(\eta)}{1 \pm d n(\eta)}\right), V_{8}=b_{1}\left(\frac{s n(\eta)}{1 \pm d n(\eta)}\right) \\
U_{9}=\sqrt{\frac{c\left(1-m^{2}\right)}{r\left(1+m^{2}\right)}-b_{1}^{2}}\left(\frac{d n(\eta)}{1 \pm m s n(\eta)}\right), V_{9}=b_{1}\left(\frac{d n(\eta)}{1 \pm m s n(\eta)}\right) \\
U_{10}=\sqrt{\frac{c\left(1-m^{2}\right)}{r\left(1+m^{2}\right)}-b_{1}^{2}}\left(\frac{s n(\eta)}{1 \pm \operatorname{sn}(\eta)}\right) \\
U_{11}=\sqrt{\frac{c}{r\left(m^{2}+1\right)}-b_{1}^{2}}(m c n(\eta) \pm d n(\eta)) \\
U_{12}=\sqrt{\frac{-c\left(1-m^{2}\right)}{r\left(1+m^{2}\right)}-b_{1}^{2}}\left(\frac{s n(\eta)}{1 \pm s n(\eta)}\right) \\
V_{13}=b_{1}\left(\frac{V_{11}}{d n(\eta) \pm c n(\eta)} b_{1}(m c n(\eta) \pm d n(\eta))\right. \\
U_{13}=\sqrt{\frac{c n(\eta)}{r\left(2-m^{2} \pm\right.}-b_{1}^{2}}\left(\frac{s n(\eta)}{\sqrt{1-m^{2} \pm d n(\eta)}}\right) \\
b_{1}\left(\frac{s n(\eta)}{d n(\eta) \pm c n(\eta)}\right)
\end{gathered}
$$

Some soliton solutions of Eq. (3.1) can be obtained in the limited case when the modulus $\mathrm{m} \rightarrow 1$ (see Appendix B)[22], as follows.

$$
\begin{aligned}
U_{1}^{1} & =\sqrt{\frac{c}{r}-b_{1}^{2}} \tanh (\eta), V_{1}^{1}=b_{1} \tanh (\eta) . \\
U_{3}^{1} & =\sqrt{\frac{2 c}{r}-b_{1}^{2}} \operatorname{sech}(\eta), V_{3}^{1}=b_{1} \sec h(\eta) . \\
U_{4}^{1} & =\sqrt{b_{1}^{2}-\frac{2 c}{r}} \operatorname{csch}(\eta), V_{4}^{1}=b_{1} \operatorname{csch}(\eta) . \\
U_{5}^{1} & =\sqrt{b_{1}^{2}-\frac{2 c}{r}} \operatorname{csch}(\eta), V_{5}^{1}=b_{1} \operatorname{csch}(\eta) .
\end{aligned}
$$

Some trigonometric-function solutions of Eq. (3.1) can be obtained in the limited case when the modulus $\mathrm{m} \rightarrow 0$ (see Appendix B). For example,

$$
\begin{gathered}
U_{1}^{2}=i b_{1} \sin (\eta), V_{1}^{2}=b_{1} \sin (\eta) . \\
U_{4}^{2}=\sqrt{\frac{2 c}{r}-b_{1}^{2}} \operatorname{csch}(\eta), V_{4}^{2}=b_{1} \operatorname{csch}(\eta) . \\
U_{5}^{2}=\sqrt{b_{1}^{2}-\frac{c}{r}} \cot (\eta), V_{5}^{2}=b_{1} \cot (\eta) .
\end{gathered}
$$




\section{Conclusions}

In this study, we have applied Auxiliary equation method to obtain the generalized solitary wave solutions of Non-Linear Klein-Gordon equation. As we can see in the example of (K. G.) equation, the main advantage of this method over the other methods is that it can be applied to a wide class of nonlinear evolution equations including those in which the odd and even-order derivative terms are coexist. It may be concluded that, Auxiliary equation method can be easily extended to all kinds of nonlinear equations.

\section{Appendix A}

\begin{tabular}{|c|c|c|c|c|}
\hline Cases & $\mathrm{R}$ & Q & $\mathrm{Au}$ & $\begin{array}{l}\text { solution of } \\
\text { uxiliary equation }\end{array}$ \\
\hline 1 & 1 & $-\left(1+m^{2}\right)$ & $\mathrm{m}^{2}$ & $\operatorname{sn}(\eta), \operatorname{cd}(\eta)$ \\
\hline 2 & $1-m^{2}$ & $2 m^{2}-1$ & $-m^{2}$ & $\operatorname{cn}(\eta)$ \\
\hline 3 & $m^{2}-1$ & $2-m^{2}$ & -1 & $\operatorname{dn}(\eta)$ \\
\hline 4 & $m^{2}\left(m^{2}-1\right.$ & $2 m^{2}-$ & -1 & $\operatorname{ds}(\eta)$ \\
\hline 5 & $1-m^{2}$ & $2-m^{2}$ & 1 & $\operatorname{cs}(\eta)$ \\
\hline & & & 2 & $s n(\eta)$ \\
\hline 6 & $\frac{1}{4}$ & $\frac{m^{2}-2}{2}$ & $\frac{m^{2}}{4}$ & $\overline{1 \pm d n(\eta)}$ \\
\hline 7 & $\frac{m^{2}}{4}$ & $\frac{m^{2}-2}{2}$ & $\frac{m^{2}}{4}$ & $\operatorname{sn}(\eta) \pm \operatorname{I} \operatorname{cn}(\eta)$ \\
\hline$d n(\eta)$ & & & & \\
\hline \multicolumn{5}{|c|}{$\overline{\mathrm{I} \sqrt{1-m^{2} \operatorname{sn}(\eta) \pm c n(\eta)}}$} \\
\hline \multirow{3}{*}{8} & \multirow{3}{*}{$\frac{1}{4}$} & \multirow{3}{*}{$\frac{1-2 m^{2}}{2}$} & \multirow{3}{*}{$\frac{1}{4}$} & $d n(\eta)$ \\
\hline & & & & $\overline{c n(\eta) \pm \mathrm{I} \sqrt{1-m^{2}}}$ \\
\hline & & & &, $\operatorname{sn}(\eta) \pm \operatorname{Idn}(\eta)$ \\
\hline \multirow{2}{*}{9} & $m^{2}-1$ & $m^{2}+1$ & $m^{2}-1$ & $d n(\eta)$ \\
\hline & 4 & 2 & 4 & $\overline{1 \pm m . c n(\eta)}$ \\
\hline 10 & $\frac{1-m^{2}}{4}$ & $\frac{m^{2}+1}{2}$ & $\underline{1-m^{2}}$ & $c n(\eta)$ \\
\hline \multirow[b]{2}{*}{11} & $-\left(1-m^{2}\right)^{2}$ & $m^{2}+1$ & & \\
\hline & $\frac{-(1-m)}{4}$ & $\frac{m+1}{2}$ & $\frac{1}{4}$ & $\mathrm{~m} \operatorname{cn}(\eta) \pm \operatorname{dn}(\eta)$ \\
\hline \multirow[b]{2}{*}{12} & & $m^{2}+1$ & $\left(1-m^{2}\right)^{2}$ & $s n(\eta)$ \\
\hline & $\frac{1}{4}$ & $\frac{m^{2}+1}{2}$ & $\frac{(1-m)}{4}$ & $\overline{d n(\eta) \pm c n(\eta)}$ \\
\hline \multirow{2}{*}{13} & $\frac{1}{4}$ & $\underline{m^{2}-2}$ & $\underline{m^{2}}$ & $c n(\eta)$ \\
\hline & & & 4 & $\overline{\mathrm{I} \sqrt{1-m^{2} \pm d n(\eta)}}$ \\
\hline
\end{tabular}

where $m$ is the modulus of the Jacobi elliptic functions which satisfies $(0 \leq m \leq 1)$.

\section{Appendix B}

The Jacobi elliptic functions degenerate as hyperbolic functions when $m \rightarrow 1$.

\begin{tabular}{|lllllc|}
\hline $\operatorname{sn}(\eta)$ & $\operatorname{cn}(\eta)$ & $\operatorname{dn}(\eta)$ & $\operatorname{sc}(\eta)$ & $\operatorname{sd}(\eta)$ & $\operatorname{cd}(\eta)$ \\
$\tanh (\eta)$ & $\operatorname{sech}(\eta)$ & $\operatorname{sech}(\eta)$ & $\sinh (\eta)$ & $\sinh (\eta)$ & 1 \\
$\operatorname{ns}(\eta)$ & $\operatorname{nc}(\eta)$ & $\operatorname{nd}(\eta)$ & $\operatorname{cs}(\eta)$ & $\operatorname{ds}(\eta)$ & $\operatorname{dc}(\eta)$ \\
$\operatorname{coth}(\eta)$ & $\cosh (\eta)$ & $\cosh (\eta)$ & $\operatorname{csch}(\eta)$ & $\operatorname{csch}(\eta)$ & 1 \\
\hline
\end{tabular}

The Jacobi elliptic functions degenerate as trigonometric functions when $m \rightarrow 0$,

$\begin{array}{cccccc}\operatorname{sn}(\eta) & \operatorname{cn}(\eta) & \operatorname{dn}(\eta) & \operatorname{sc}(\eta) & \operatorname{sd}(\eta) & \operatorname{cd}(\eta) \\ \sin (\eta) & \cos (\eta) & 1 & \tan (\eta) & \sin (\eta) & \cos (\eta) \\ \operatorname{ns}(\eta) & \operatorname{nc}(\eta) & \operatorname{nd}(\eta) & \operatorname{cs}(\eta) & \operatorname{ds}(\eta) & \operatorname{dc}(\eta) \\ \csc (\eta) & \sec (\eta) & 1 & \cot (\eta) & \operatorname{csch}(\eta) & \sec (\eta)\end{array}$

\section{REFERENCES}

[1] MJ. Ablowitz, H. Segur. Philadelphia: SIAM; 1981 .

[2] VO. Vakhnenk, EJ. Parke, AJ. Morrison. Chaos, Solitons \& Fractals 2003;17(4):683 92.

[3] R. Hirota. Springer;1980.p.115775.

[4] W. Malfliet. Am J. Phys 1992;60:650--- 4.

[5] AM.Wazwaz. Math Comput Modelling 2004;40:499 508.

[6] E.Fan, H.Zhang. Phys. Lett 1998;246:403-6.

[7] F.Caloger, A.Degasperis. Nuovo Cimento B 32, 201, (1976).

[8] JH. He, MA. Abdou. Chaos, Solitons \& Fractals 2007;34:14219.

[9] O. Bogoyavlenskii. Usp. Mat. Nauk. 45, 17, (1990).

[10] Chen Huai-Tang, Zhang Hong-Qing. Chaos, Solitons and Fractals 20 (2004) 765-769.

[11] A. A. Halim, S. B. Leble. Chaos, Solitons and Fractals 19 (2004) 99-108.

[12] A. A. Halim, S. B. Leble. Chaos, Solitons and Fractals 19 (2004) 99-108.

[13] JH. He, MA. . Chaos, Solitons \& Fractals 2007;34:14219.

[14] Chen Huai-Tang, Zhang Hong-Qing,. Chaos, Solitons and Fractals 20 (2004) 765.769.

[15] P.G. Kevrekidis, V.V. Konotop, Math. Comput. Simulat. 62 (2003) 79.89

[16] P.G. Kevrekidis, V.V. Konotop, Math. Comput. Simulat. 62 (2003) 79.89.

[17] S. Nakagiri, J. Ha, Nonlinear Anal. 47 (2000) 89.100.

[18] R. Temam. Applied Math. Sci., 68, Springer-Verlag, 1988.

[19] H. Fusaoka, J. Phys. Soc., Japan 59 (2) (1990) 455.463.

[20] D. Kaya, S.M. El-Sayed, Applied Mathematics and Computation, (2003) in press.

[21] I.J. Lee, J. Korean Math. Soc. 32 (3) (1995) 541.551.

[22] M.H.M. Moussa, Rehab M. El-Shiekh. Intern. J. Nonlinear Sci. Vol.10(2010)No. 1, pp70-76. 